\title{
ELECCIONES DE 28 DE OCTUBRE DE 1990 AL
}

PARLAMENTO VASCO

CARMEN FERNÁNDEZ-MIRANDA CAMPOAMOR

Profesora Titular de Derecho Constitucional

UNED 


\section{SUMARIO}

I. Campaña electoral.-II. Jornada electoral.-1. Abstención. 2. Resultados en las tres provincias del País Vasco.

3. Valoración. a) Voto nacionalista. b) Voto españolista.

c) Posibles coaliciones de Gobierno. 
Revista de Derecho Político, núm. 33, 1991, pp. 263-291

\title{
ELECCIONES DE 28 DE OCTUBRE DE 1990 AL PARLAMENTO VASCO
}

\author{
POR \\ CARMEN FERNÁNDEZ-MIRANDA CAMPOAMOR \\ Profesora Titular de Derecho Constitucional \\ UNED
}

\begin{abstract}
El 28 de octubre de 1990 se celebran en el País Vasco las cuartas elecciones autonómicas. El cuerpo electoral consta de 1.680 .929 ciudadanos que elegirán los 75 escaños que forman el Parlamento de Vitoria, correspondiendo 25 a cada provincia, con lo que Álava resulta hiperrepresentada '. En el siguiente cuadro se muestran los partidos políticos contendientes, su candidato a lendakari y los escaños que tienen desde la consulta de 1986.
\end{abstract}

\section{CAMPAÑA ELECTORAL}

En esta campaña, caracterizada por la normalidad, existe la opinión mayoritaria de que en el periodo $1986-90$ se ha producido una importante mejoria política y económica en Euskadi, y, en consecuencia, un clima de tranquilidad y esperanza inhabitual en los últimos años. La sociedad vasca da muestras de cansancio ante la violencia, y la necesidad de paz comienza a ser una obsesión ciudadana ${ }^{2}$.

1 En Álava se necesitan 5.600 votos para lograr un escaño, en Guipúzcoa 14.900 y 25.600 en Vizcaya. Datos aportados en Diario 16 de 2 de noviembre de 1990. “Análisis post-electorales». Belén Diez Caballero.

2 Lo que la gente quiere es que todo deje de terminar violentamente, desde una huelga đé autobuses a una conversación. Declaraciones del periodista Luciano Rincón, recogidas por El País de 28 de octubre de 1990. 
CUADRO 1

\begin{tabular}{|c|c|c|}
\hline SIGLAS & $\begin{array}{l}\text { PARTIDOS POLITICOS } \\
\text { (CANDIDATO A LENDAKARI) }\end{array}$ & $\begin{array}{l}\text { ESCAÑOS } \\
1986-90\end{array}$ \\
\hline EAJ-PNV & $\begin{array}{l}\text { Eusko Alderdi Yelzalea-Partido Nacionalista Vasco } \\
\qquad \text { (J. A. Ardanza) }\end{array}$ & 17 \\
\hline PSE-PSOE & Partido Socialista de Euskadi (R. Jáuregui) ............... & 19 \\
\hline HB & Herri Batasuna (J. Idigoras) ............. & 13 \\
\hline EA & Eusko Alkartasuna (C. Garaicoetxea) ............................ & 13 \\
\hline EE & Euskadiko Ezquerra (Kepa Aulestia) & 9 \\
\hline CDS & Centro Democrático y Social (A. Marco Tobar) ........ & 2 \\
\hline PP & Partido Popular (J. Mayor Oreja) & 2 \\
\hline DS & Democracia Socialista (Garcia Damborenea) .... & - \\
\hline UA & Unidad Alavesa (J. L. Añua) & - \\
\hline
\end{tabular}

La propuesta más espectacular es la realizada por el candidato de Herri Batasuna, Iñaqui Esnaola, al presentar un documento en el que manifiesta el ofrecimiento de su partido a formar un gobierno abertzale con el Partido Nacionalista Vasco e Eusko Alkartasuna (sin contar con Euskadiko Ezquerra).

Sus condiciones son claras y terminantes:

- Aprobación de un programa politico en el que se sentarán las bases para resolver los problemas de fondo generados por el enfrentamiento Euskadi-Estado.

- Reconocimiento del derecho a la autodeterminación.

- Nuevo marco jurídico-político con el euskera como lengua nacional.

— Unidad territorial. 
Si se logra tal pacto «HB no escatimará esfuerzos para la desaparición de la violencia, con una premisa: la negociación entre ETA y el Estado español como único medio para lograr solucionar el problema de la soberanía política» ${ }^{3}$.

Este ofrecimiento, aceptado con entusiasmo por los militantes abertzales, genera desconfianza y sospecha en la mayoria de la población que se pregunta cómo HB no intenta, si es que está en sus manos, acabar con la violencia sin tantas condiciones previas. Entre los nacionalistas democráticos la oferta de HB genera toda clase de rechazos directos y expresos, exigiendo la renuncia a la violencia como punto de partida para cualquier negociación y negándose a todo pacto con un partido que no reconoce la legitimidad de las instituciones vascas, ni acude a ellas a presentar sus reivindicaciones politicas.

El interés de la campaña, convencido el electorado de la imposibilidad de que ninguna formación política logre la mayoría absoluta, se centra en la valoración, como opción de futuro, de la continuidad del pacto Partido Nacionalista Vasco-Partido Socialista, o de la aceptación de una coalición nacionalista, Partido Nacionalista, Eusko Alkartasuna y Euskadiko Ezquerra, que suponga la mayoría natural vasquista, con la única exclusión del abertzalismo antisistema, brazo político de ETA ${ }^{4}$. EA y EE animan al electorado a respaldar el pacto nacionalista que estaría alejado «tanto del centralismo que amordaza el nacionalismo moderado, como del nacionalismo violento que respalda a ETA" ${ }^{5}$. El Partido Socialista, por su parte, pretende hacer llegar a la población tres ideas fundamentales. En primer lugar, presenta su pacto con el PNV como la causa de la bonanza política y económica lograda en Euskadi en los últimos cuatro años, al tener «unos interlocutores cualificados en el Gobierno de Madrid"; es decir, estableciendo una relación directa entre la mejoría notoria de la sociedad vasca y la coincidencia de una de las partes de la coalición con el partido que gobierna el Estado. En segundo lugar, el rechazo a la coalición nacionalista - pese a su aceptación sentimental de parte de la sociedad- porque originaría continuas tensiones entre los firmantes, al tener un terreno electoral común $-y$ continuas tensiones con el Gobierno central ante la divergencia de planteamientos en numerosas cuestiones. Por último, y como resumen de las otras dos, porque toda recuperación económica exige estabilidad política ${ }^{6}$.

$3 \quad A B C$, El Mundo, Diario 16, El Pais del 21 de octubre de 1990.

${ }_{4}$ En los mítines de HB sigue oyéndose el cuarto grito de "Gora eta militarra", con lo que supone de provocación.

5 Declaraciones de C. Garaicoetxea, recogidas por Diario 16 de 23 de octubre de 1990. Sin embargo, según avanza la campaña, las preferencias del electorado se centran en el pacto PNV-PSOE. Ver gráficas de sondeos.

${ }^{5}$ Declaraciones de R. Jáuregui recogidas en Diario 16 y El País de 25 de octubre de 1990. 
El Partido Nacionalista Vasco, convencido de la aceptación de su gestión de gobierno, pretende aumentar sus escaños para poder dirigir la negociación previa al pacto de gobierno - sea cual fuere el que prefiera el electorado- A su vez, el Partido Popular intenta consolidar su presencia en Euskadi, reacia en los últimos tiempos a votar a la derecha española, demasiado antinacionalista. El Centro Democrático y Social busca no desaparecer del Parlamento de Vitoria, al que quiere llegar, por su parte, Unión Alavesa, para reivindicar, en el foro adecuado, las peculiaridades de su provincia. Los sondeos de opinión realizados durante la campaña dan los siguientes datos ${ }^{\text {? }}$ :

7 Datos obtenidos por el Instituto Demascopia y publicados en El Pais del 21 de octubre de 1990. 
CUADRO 2

VOTO PROBABLE Y REPARTO DE ESCAÑOS

(Porcentaje sobre el total de votos válidos)

\begin{tabular}{|c|c|c|c|c|c|c|c|c|c|c|c|c|c|c|c|c|}
\hline \multirow{3}{*}{$\begin{array}{l}\text { PARTIDOS } \\
\text { POLITICOS } \\
\text { (SIGLAS) }\end{array}$} & \multicolumn{4}{|c|}{ PAIS VASCO } & \multicolumn{4}{|c|}{ ALAVA } & \multicolumn{4}{|c|}{ GUIPÚZCOA } & \multicolumn{4}{|c|}{ VIZCAYA } \\
\hline & \multicolumn{2}{|c|}{1990} & \multicolumn{2}{|c|}{1986} & \multicolumn{2}{|c|}{1990} & \multicolumn{2}{|c|}{1986} & \multicolumn{2}{|c|}{1990} & \multicolumn{2}{|c|}{1986} & \multicolumn{2}{|c|}{1990} & \multicolumn{2}{|c|}{1986} \\
\hline & $\begin{array}{c}\% \\
\text { votos }\end{array}$ & $\begin{array}{c}\text { Esca- } \\
\text { ños }\end{array}$ & $\begin{array}{c}\% \\
\text { votos }\end{array}$ & $\begin{array}{l}\text { Esca- } \\
\text { ños }\end{array}$ & $\begin{array}{c}\% \\
\text { votos }\end{array}$ & $\begin{array}{c}\text { Esca- } \\
\text { ños }\end{array}$ & $\begin{array}{c}\% \\
\text { votos }\end{array}$ & $\begin{array}{c}\text { Esca- } \\
\text { ños }\end{array}$ & $\begin{array}{c}\% \\
\text { votos }\end{array}$ & $\begin{array}{c}\text { Esca- } \\
\text { ños }\end{array}$ & $\begin{array}{c}\% \\
\text { votos }\end{array}$ & $\begin{array}{c}\text { Esca- } \\
\text { ños }\end{array}$ & $\begin{array}{c}\% \\
\text { votos }\end{array}$ & $\begin{array}{c}\text { Esca- } \\
\text { ños }\end{array}$ & $\begin{array}{c}\% \\
\text { votos }\end{array}$ & $\begin{array}{r}\text { Esca } \\
\text { ños }\end{array}$ \\
\hline PNV-EAJ & 27,6 & $21-23$ & 23,7 & 17 & 25,9 & $7-8$ & 20,2 & 5 & 19,8 & $5-6$ & 16,0 & 4 & 32,6 & 9 & 28,9 & 8 \\
\hline EA & 10,6 & $7-9$ & 15,9 & 13 & 7,2 & $1-2$ & 14,6 & 4 & 19,0 & 5 & 23,3 & 6 & 6,6 & $1-2$ & 11,8 & 3 \\
\hline $\mathrm{HB}$ & 17,4 & $12-14$ & 17,5 & 13 & 10,2 & $2-3$ & 12,9 & 3 & 22,7 & $6-7$ & 21,7 & 6 & 15,9 & 4 & 16,0 & 4 \\
\hline $\mathrm{EE}$ & 8,2 & 7 & 10,9 & 9 & 10,6 & 3 & 11,0 & 3 & 8,5 & 2 & $\uparrow 1,9$ & 3 & 7,6 & 2 & 10,3 & 3 \\
\hline PSE-PSOE & 20,8 & $17-20$ & 22,0 & 19 & 26,0 & $7-8$ & 25,0 & 7 & 19,6 & $5-6$ & 20,0 & 6 & 20,2 & $5-6$ & 22,6 & 6 \\
\hline PP & 9,7 & $6-8$ & 4,8 & 2 & 13,2 & $3-4$ & 6,8 & 1 & 5,4 & $0-1$ & 3,6 & - & 11,4 & 3 & 5,2 & 1 \\
\hline CDS $\ldots \ldots \ldots \ldots \ldots \ldots \ldots \ldots \ldots$ & 2,8 & 一 & 3,5 & 2 & 2,6 & 一 & 8,1 & 2 & 2,2 & - & 2,4 & - & 3,2 & - & 3,2 & 一 \\
\hline UNIDAD ALAVESA .. & 0,3 & 一 & 一 & 一 & 2,1 & 一 & 一 & - & 一 & - & 一 & 一 & 一 & 一 & - & - \\
\hline OTROS & 2,6 & 一 & 1,7 & - & 2,2 & 一 & 1,4 & - & 2,8 & 一 & 1,1 & 一 & 2,5 & - & 2,0 & - \\
\hline TOTAL & 100 & 75 & 100 & 75 & 100 & 25 & 100 & 25 & 100 & 25 & 100 & 25 & 100 & 25 & 100 & 25 \\
\hline
\end{tabular}




\section{CUADRO 3}

¿QUIÉN LE GUSTARÍA A USTED QUE FUESE EL PRÓXIMO LENDAKARI DEL GOBIERNO VASCO?

(TOTAL DEL PAIS VASCO)

(Porcentajes)

\begin{tabular}{|c|c|c|c|c|}
\hline CANDIDATOS & TOTAL & ALAVA & GUIPÚZCOA & VIZGAYA \\
\hline 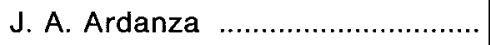 & $35 \%$ & $48 \%$ & $30 \%$ & $35 \%$ \\
\hline 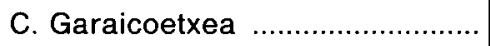 & $10 \%$ & $10 \%$ & $12 \%$ & $9 \%$ \\
\hline J. Idígoras ....... & $7 \%$ & $5 \%$ & $11 \%$ & $5 \%$ \\
\hline Kepa Aulestia ............. & $4 \%$ & $7 \%$ & $4 \%$ & $4 \%$ \\
\hline R. Jáuregui ..... & $6 \%$ & $9 \%$ & $6 \%$ & $5 \%$ \\
\hline J. Mayor Oreja & $1 \%$ & $2 \%$ & $1 \%$ & $0,3 \%$ \\
\hline A. Marco Tobar ... & $0,4 \%$ & $1 \%$ & $0,3 \%$ & $0,2 \%$ \\
\hline
\end{tabular}

\section{CUADRO 4}

VALORACIÓN DE LA SITUACIÓN ECONÓMICA Y POLITICA DE EUSKADI RESPECTO A HACE UNOS AÑOS

(Porcentajes)

\begin{tabular}{|c|c|c|}
\hline VALORACIÓN & SITUACION ECONOMICA & SITUACIÓN POLITICA \\
\hline Mejor & $35 \%$ & $37 \%$ \\
\hline 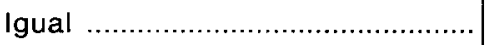 & $29 \%$ & $38 \%$ \\
\hline Peor & $30 \%$ & $15 \%$ \\
\hline NS/NC & $6 \%$ & $10 \%$ \\
\hline
\end{tabular}


CUADRO 5

EVALUACIÓN DE LA SITUACIÓN POLÍTICA DE EUSKADI EN 1986 Y 1990

(Porcentajes)

\begin{tabular}{|c|c|c|}
\hline SITUACIÓN POLITICA & NOVIEMBRE 1986 & SEPTIEMBRE-OCTUBRE 1990 \\
\hline 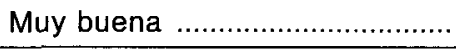 & $1 \%$ & $1 \%$ \\
\hline 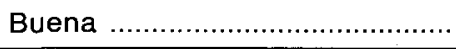 & $5 \%$ & $15 \%$ \\
\hline Regular .................... & $41 \%$ & $51 \%$ \\
\hline 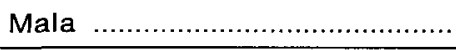 & $32 \%$ & $19 \%$ \\
\hline 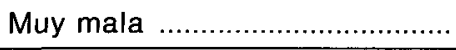 & $14 \%$ & $4 \%$ \\
\hline NS/NC & $7 \%$ & $11 \%$ \\
\hline
\end{tabular}

CUADRO 6

ACTITUD RESPECTO A UNA POSIBLE COALICIÓN DESPUÉS DE LAS ELECCIONES

(Porcentajes)

\begin{tabular}{|c|c|c|}
\hline PARTIDOS & 7. - OCTUBRE - 1990 & 21 - OCTUBRE - 1990 \\
\hline PNV-PSOE $\ldots \ldots \ldots \ldots \ldots \ldots \ldots \ldots \ldots \ldots \ldots \ldots \ldots \ldots \ldots \ldots \ldots \ldots \ldots \ldots$ & $16 \%$ & $22 \%$ \\
\hline PNV-EA-EE $\ldots \ldots \ldots \ldots \ldots \ldots \ldots \ldots \ldots \ldots \ldots \ldots \ldots \ldots \ldots \ldots \ldots \ldots$ & $16 \%$ & $18 \%$ \\
\hline PNV-EA-EE-HB & $19 \%$ & $17 \%$ \\
\hline OTRA $\ldots \ldots \ldots$ & $6 \%$ & $5 \%$ \\
\hline NS $\ldots$. & $43 \%$ & $38 \%$ \\
\hline
\end{tabular}


CUADRO 7

CONOCIMIENTO DEL EUSKERA ENTRE LOS VOTANTES

DEL PAÍS VASCO

(Porcentajes)

\begin{tabular}{|c|c|c|}
\hline CONOCIMIENTO DEL EUSKERA & 1986 & 1990 \\
\hline Lo hablan normalmente .................. & $24 \%$ & $26 \%$ \\
\hline Lo entienden pero no lo hablan normalmente & $17 \%$ & $12 \%$ \\
\hline Sólo entienden y hablan castellano & $43 \%$ & $52 \%$ \\
\hline NC ..... & $16 \%$ & $10 \%$ \\
\hline
\end{tabular}

II. JORNADA ELECTORAL

Con una baja participación del 61,13 por 100 y un clima de normalidad ciudadana, se desarrolla la jornada electoral del 28 de octubre, arrojando los siguientes resultados, ya anunciados por las encuestas, salvo en lo relativo al triunfo electoral de Unidad Alavesa que logra tres escaños ${ }^{8}$. EI Partido Nacionalista Vasco y el Partido Popular logran un claro ascenso; Herri Batasuna se mantiene, aunque pierde votos; Eusko Alkartasuna, Euskadiko Ezquerra y el Partido Socialista descienden en número de representantes, mientras que el Centro Democrático y Social es eliminado del Parlamento de Vitoria; Izquierda Unida y Democracia Socialista no logran incorporarse a la Cámara. 1990.

B Escindida del Partido Popular unos meses antes, se inscribe en enero de 


\begin{tabular}{|c|c|c|c|c|c|c|c|c|c|c|c|c|}
\hline \multirow{3}{*}{$\begin{array}{l}\text { Resultados globales } \\
\text { PARTIDOS O CANDIDATURAS }\end{array}$} & \multicolumn{12}{|c|}{ CENSO: 1.680 .929 / PARTICIPACIÓN: $61,13 \%$ / VOTOS ESCRUTADOS: $100 \%$ / VOTOS VÁLIDOS: 1.021 .963 / VOTOS EN BLANCO: 7.780 / VOTOS NULOS: 5.638} \\
\hline & \multicolumn{3}{|c|}{ Autonómicas 1990} & \multicolumn{3}{|c|}{ Autonómicas 1986} & \multicolumn{3}{|c|}{ Autonómicas 1984} & \multicolumn{3}{|c|}{ Autonómicas 1980} \\
\hline & Votos & $\%$ & Escaños & Votos & $\%$ & Escaños & Votos & $\%$ & Escaños & Votos & $\%$ & Escaños \\
\hline \multirow{11}{*}{$\begin{array}{l}\text { Partido Nacionalista } \\
\text { Vasco - EAJ } \\
\text { Partido Socialista de } \\
\text { Euskadi - PSOE } \\
\text { Herri Batasuna } \\
\text { Eusko Alkartasuna } \\
\text { Euskadiko Ezquerra } \\
\text { Partido Popular (CP) } \\
\text { Unidad Alavesa } \\
\text { Centro Democrático Social } \\
\text { Izquierda Unida } \\
\text { Democracia Socialista } \\
\text { Otros }\end{array}$} & & & & & & & & & & & & \\
\hline & 289.152 & 28,51 & 22 & 271.208 & 23,70 & 17 & 452.178 & 41,70 & 32 & 349.896 & 37,96 & 25 \\
\hline & 201.608 & 19,87 & 16 & 252.233 & 22,04 & 19 & 247.786 & 23,30 & 19 & 130.484 & 14.14 & 9 \\
\hline & 186.277 & 18,36 & 13 & 199.900 & 17,47 & 13 & 157.389 & 14,61 & 11 & 152.097 & 16,49 & 11 \\
\hline & 115.755 & 11,41 & 9 & 181.175 & 15,83 & 13 & & & & & & \\
\hline & 78.855 & 7,77 & 6 & 124.423 & 10,87 & 9 & 85.671 & 8,02 & 6 & 90.905 & 9,76 & 6 \\
\hline & 83.482 & 8,23 & 6 & 55.606 & 4,86 & 2 & 100.581 & 9,39 & 7 & 43.853 & 4,75 & 2 \\
\hline & 14.373 & $\uparrow, 41$ & 3 & & & & & & & & & \\
\hline & 6.652 & 0,65 & & 40.445 & 3,53 & 2 & & & & 78.310 & 8,46 & $6^{1}$ \\
\hline & 14.272 & 1,40 & & 6.750 & 0,60 & & & & & 37.051 & 4,01 & $1^{2}$ \\
\hline & 5.000 & 0,49 & & & & & & 200 & & & & \\
\hline TOTAL ESCANOS & & & 75 & & & 75 & & & & & & 60 \\
\hline
\end{tabular}

' Los datos corresponden a los resultados obtenidos por UCD, Unión de Centro Democrático.

${ }^{2}$ Los datos corresponden a los resultados obtenidos por PCE-EPK, Partido Comunista de Euskadi. 


\title{
1. Abstención
}

\author{
CUADRO $9^{10}$
}

(Porcentajes)

\begin{tabular}{|l|c|c|c|c|c|c|c|}
\hline \multicolumn{1}{|c|}{ ELECCIONES } & 1979 & 1980 & 1983 & 1984 & 1986 & 1987 & 1990 \\
\hline Autonómicas & & $45 \%$ & & $32,8 \%$ & $29,4 \%$ & & $38,87 \%$ \\
\hline Municipales & $30,6 \%$ & & $35 \%$ & & & $32,27 \%$ & \\
\hline
\end{tabular}

Baja participación sólo superada por las elecciones autonómicas de 1980, quizá la normalización económica y política de la sociedad vasca sea, en este caso, la causa de la abstención registrada. Es, por tanto, el desinterés que afecta al ciudadano, cansado de tanta consulta electoral, cuando no considera que su voto vaya a ser decisorio, sino un voto más en una situación estabilizada; sólo en estos casos la meteorología puede coadyuvar al abandono ${ }^{11}$, abandono que beneficia claramente a los partidos extremistas cuyos votantes siempre acuden a las urnas. El resultado es, pues, una alta abstención que, unida al 17,47 por 100 de votos logrados por Herri Batasuna, convierte en preocupante la posibilidad de lograr una definitiva estabilidad de las sociedad vasca, ante un 55,34 por 100 del electorado que no respalda expresamente el pacto de los partidos democráticos $^{12}$.

\section{Resultados en las tres provincias del Pais Vasco}

En Alava vence el Partido Nacionalista Vasco en número de votos, aunque logra igual número de escaños que el Partido Socialista; sorpresa de Unión Alavesa que logra tres representantes en su provincia. Clara victoria del Partido Nacionalista Vasco en su feudo tradicional de Vizcaya, con 10 escaños frente a los cinco obtenidos por el Partido Socialista. Triunfo de Herri Batasuna en Guipúzcoa en número de votos, aunque obtiene igual representación que el Partido Nacionalista Vasco.

10 Datos recogidos por El País, 29-X-90.

1 El mal tiempo reinante fue utilizado por los perdedores como causa directa de su fracaso.

12 Diario 16, 29-X-90. Lourdes Ortiz. 


\section{CUADRO $10^{13}$}

\section{ÁLAVA}

Electores: 211.727

Parlamentarios: 25.

$\%$ escrutado: 100.

Nulos y Blanco: 1.872 .

\begin{tabular}{|c|c|c|c|c|c|c|}
\hline \multirow[b]{2}{*}{$\begin{array}{c}\text { PARTIDOS } \\
\text { (SIGLAS) }\end{array}$} & \multicolumn{3}{|c|}{ ELECCIONES 90} & \multicolumn{3}{|c|}{ ELECCIONES 86} \\
\hline & Votos & $\%$ & Escaños & Votos & $\%$ & Escaños \\
\hline PNV & 28.371 & 22,56 & 6 & 27.975 & 20,15 & 5 \\
\hline PSE-PSE & 26.755 & 21,26 & 6 & 27.975 & 25,03 & 7 \\
\hline $\mathrm{HB}$ & 16.057 & $12 ; 77$ & 3 & 20.248 & 14,58 & 4 \\
\hline UA & 14.039 & 11,16 & 3 & 17.860 & 12,86 & 3 \\
\hline${ }^{*} P P$ & 13.745 & 10,93 & 3 & 15.256 & 10,99 & 3 \\
\hline EA & 10.316 & 8,20 & 2 & 9.428 & 6,79 & 1 \\
\hline EE & 8.467 & 6,73 & 2 & - & - & 一 \\
\hline
\end{tabular}

* (En el 86, Coalición Popular).

PNV: José Antonio Ardanza Garro, Félix Ormazábal Ascasibar, Juan Maria Ollora Ochoa de Aspuru, Juan José Ibarreche Marcuartu, Maria Isabel Orbe Garay y Alvaro Amman Rabanera. PSE-PSOE: Ramón Jáuregui Atondo, Fernando Buesa Blanco, Julio Herrero Romero, Maria Blanca Elena Alday Carrasco, Alfonso Martínez Pérez y Roberto San Ildefonso Izaguirre. HB: Iñaki Ruiz de Pinedo Undiano, Pablo Gorotiaga González y Xabier Elorriaga Preciado. EA: Patxi Ormazábal Zamakona y Rafael Larreina Valderrama. EE: Pablo Ruiz de Gordejuela Urquijo y Xabier Markiegi Candina. PP: Jaime Mayor Oreja, Enrique Villar Montero y Carmelo Barrio Baroja. UA: José Luis Añua Ajuria, María Enriqueta Benito Bengoa y Maria Teresa Mendaza Garcia de Vicuña.

13 Datos oficiales publicados por Diario 16, 29-X-90. 


\section{GUIPÚZCOA}

Electores: 540.887

Parlamentarios: 25.

$\%$ escrutado: 100.

Nulos y Blanco: 4.117 .

\begin{tabular}{|c|c|c|c|c|c|c|}
\hline \multirow[b]{2}{*}{$\begin{array}{l}\text { PARTIDOS } \\
\text { (SIGLAS) }\end{array}$} & \multicolumn{3}{|c|}{ ELECCIONES 90} & \multicolumn{3}{|c|}{ ELECCIONES 86} \\
\hline & Votos & $\%$ & Escaños & Votos & $\%$ & Escaños \\
\hline $\mathrm{HB}$ & 79.288 & 23,8 & 6 & 86.909 & 23,31 & 6 \\
\hline PNV & 68.361 & 20,5 & 6 & 80.926 & 21,70 & 6 \\
\hline PSE-PSOE $\ldots \ldots \ldots \ldots \ldots$ & 63.358 & 19,5 & 5 & 74.466 & 19,97 & 6 \\
\hline EA & 60.459 & 18,1 & 5 & 59.653 & 16,00 & 4 \\
\hline ............... & 29.599 & 8,9 & 2 & 44.418 & 11,91 & 3 \\
\hline${ }^{*} \mathrm{PP}$. & 21.580 & 6,4 & 1 & 13.324 & 3,57 & - \\
\hline IU-EB ..... & 3.319 & 0,9 & 一 & - & - & - \\
\hline
\end{tabular}

* (En el 86, Coalición Popular).

PNV: Joseba Arregui Aranburu, Joseba Leizaola Azpiazu, Esther Larrañaga Aguirre, Joseba Egíbar Artola y Luis María Bandrés Unanue, José Manuel Martiarena Lizarazu. PSE-PSOE: Jesús Eguiguren Imaz, José Antonio Maturana Plaza, José Ramón Recalde Díez, Odón Elorza González y Manuel Huertas Vicente. HB: Iñaki Esnaola Etxeberri, Iñigo Iruin Sanz, Rafael Fíez Usabiaga, Maitane Intxaurraga Uribarri, Jokin Gorotidi Artola y José Maria Elosua Sánchez. EA: Carlos Garaikoetxea Urriza, Juan Porres Azcona, Esther Larrañaga Galdós, Mikel Olaciregui Martínez de llarduya y Federico Abaicar Marticorena. EE: Xabier Gurrutxaga Aizpeolea y Koro Agote Ugartemendia. PP: Gregorio Ordóñez Fenollar. 
VIZCAYA

Electores: 928.315

Parlamentarios: 25.

$\%$ escrutado: 100.

Nulos y Blanco: 7.390 .

\begin{tabular}{|c|c|c|c|c|c|c|}
\hline \multirow[b]{2}{*}{$\begin{array}{l}\text { PARTIDOS } \\
\text { (SIGLAS) }\end{array}$} & \multicolumn{3}{|c|}{ ELECCIONES 90} & \multicolumn{3}{|c|}{ ELECCIONES 86} \\
\hline & Votos & $\%$ & Escaños & Votos & $\%$ & Escaños \\
\hline PNV & 192.420 & 34,6 & 10 & 183.365 & 28,91 & 8 \\
\hline PSE-PSE & 111.495 & 20,05 & 5 & 143.231 & 21,70 & 6 \\
\hline HB & 90.932 & 16,3 & 4 & 101.636 & 16,02 & 4 \\
\hline EA & 44.980 & 8,08 & 2 & 74.481 & 11,80 & 3 \\
\hline $\mathrm{EE}$ & 40.789 & 7,33 & 2 & 65.048 & 10,25 & 3 \\
\hline${ }^{*} \mathrm{PP}$ & 48.157 & 8,56 & 2 & 32.730 & 5,16 & 1 \\
\hline IU-EB & 9.523 & 1,7 & - & - & - & - \\
\hline
\end{tabular}

* (En el 86, Coalición Popular).

PNV: José Antonio Rubalcaba Quintana, Xabier Aguirre Bilbao, Idoya Zemarruzabeitia Beldarrain, Txomin Aurrekoetxea Iza, Joseba Andoni Aurrecoechea Vergara, Carmelo Sainz-Maza Arrola, Yolanda Sangroniz Aguirrebeitia, Xabier Ormaetxea Garay, Pedro Luis Urrutxi Arnoriaga y Maria Isabel Juaristi Torrealday. PSE-PSOE: Nicolás Redondo, José Andrés Paul Tejedor, Luis María Atienza Serna, Rosa María Díez González y Jesús María Rodríguez Orrantia. HB: Taxio Erkizia Almandoz, Jone Goirizelai Ordorika, Iñaki Antigüedad Auzmendi y Gorka Martinez Bilbao. EA: Juan Daniel Barandiarán Jaca y Sabín Intxaurraga Mendibil. EE: Kepa Aulestia Urrutia y Xabier Garmendia Martínez. PP: Leopoldo Barreda de los Rios y Fernando Maura Barandiarán. 


\section{CUADRO $11^{14}$}

\section{GUIPÚZCOA, PUEBLO A PUEBLO}

Estos son los resultados definitivos de las localidades más importantes de la provincia de Guipúzcoa:

- Aya. PSOE: 10; PNV: 367; EE: 16; PP: 2; EA: 180; HB: 261.

- Andoaín. PSOE: 1.840; HB: 1.725; PNV: 1.234; EE: 799; PP: 284; EA: 1.086.

- Arechabaleta. PSOE: 525; PNV: 713; HB: 948; PP: 23; EE: 135; EA: 727.

- Azpeitia. PSOE: 420; PNV: 2.326; EE: 349; PP: 240; EA: 2.041; HB: 1.250.

- Beasaín. HB: 1.405; PSOE: 1.200; PNV: 1.467; EE: 672; PP: 240; EA: 1.122.

- Elgoibar. PSOE: 1.094; PNV: 2.223; EE: 559; PP: 173; EA: 883; HB: 1.248.

- Hernani. PSOE: 1.743; PNV: 1.182; EE: 700; PP: 236; EA: 1.301; HB: 3.246.

- Irún. PSOE: 7.221; PNV: 3.740; EE: 2.558; PP: 2.275; EA: 3.124; HB: 3.523.

- Lascao. PSOE: 435; PNV: 688; EE: 212; PP: 34; EA: 389; HB: 822.

- Legazpia. PSOE: 1.012; PNV: 1.113; EE: 667; PP: 162; EA: 1.309; HB: 1.082.

- Motrico. PSOE: 131; PNV: 1.340; EE: 92; PP: 130; EA: 234; HB: 834.

- Oñate. PSOE: 461; PNV: 2.264; EE: 433; PP: 188; EA: 882; HB: 1.509.

- Pasajes. PSOE: 1.890; PNV: 1.168; EE: 777; PP: 307; EA: 1.311; HB: 2.532.

- Renteria. PSOE: 6.070; PNV: 2.047; EE: 1.834; PP: 761; EA: 2.240; HB: 4.813.

- San Sebastián. PSOE: 15.113; PNV: 14.063; EE: 8.521; PP: 11.160; EA: 15.102; HB: 15.747 .

$14 \quad A B C, 29-X-90$. 
- Tolosa. PSOE: 984; PNV: 1.971; EE: 824; PP: 708; EA: 1.897; HB: 2.237.

- Urrecho. PSOE: 803; PNV: 541; EE: 301; PP: 55; EA: 980; HB: 648.

- Vergara. HB: 2.683; PSOE: 983; PNV: 2.164; EE: 590; PP: 227; EA: 1.277.

- Villabona. PSOE: 358; PNV: 423; EE: 354; PP: 49; EA: 528; HB: 873.

- Zarauz. PSOE: 1.090; PNV: 1.787; EE: 911; PP: 567; EA: 2.475; HB: 1.620.

- Zumárraga. PSOE: 1.740; PNV: 998; EE: 569; PP: 136; EA: 1.298; HB: 797.

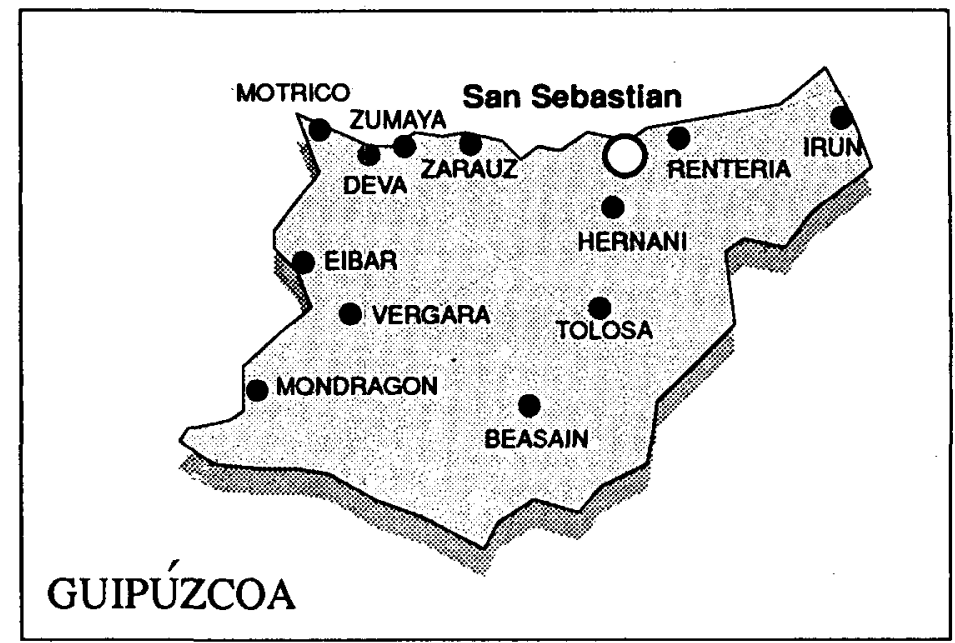


CUADRO 12

VIZCAYA, PUEBLO A PUEBLO

Los votos obtenidos por los principales partidos políticos en algunas ciudades de la circunscripción de Vizcaya, según los resultados al 100 por $100 \mathrm{del}$ escrutinio son los siguientes:

- Amorebieta. PNV: 3.105; HB: 2.000; PSOE: 1.414; EA: 821; PP: 185; EE: 440.

- Arrigorriaga.'PNV: 1.669; HB: 950; PSOE: 949; EA: 227; PP: 123; EE: 386.

- Balmaseda. PNV: 1.622; HB: 550; PSOE: 1.042; EA: 448; PP: 224; EE: 156.

- Baracaldo. PNV: 12.780; HB: 6.902; PSOE: 14.933; EA: 3.055; PP: 3.821; EE: 3.669 .

- Basauri. PNV: 7.072; HB: 3.650; PSOE: 6.252; EA: 1.088; PP: 1.252; EE: 1.698.

- Bilbao. PNV: 62.807; HB: 23.382; PSOE: 35.842; EA: 10.950; PP: 23.150; EE: 14.627 .

- Bermeo. PNV: 3.127; HB: 2.218; PSOE: 659; EA: 2.580; PP: 132; EE: 500.

- Durango. PNV: 4.397; HB: 2.016; PSOE: 1.620; EA: 902; PP: 1.100; EA: 723.

- Erandio. PNV: 3.930; HB: 1.790; PSOE: 2.614; EA: 731; PP: 370; EE: 810.

- Galdácano. PNV: 4.182; HB: 2.612; PSOE: 2.491; EA: 1.464; PP: 547; EE: 803.

- Guecho. PNV: 14.428; HB: 4.974; PSOE: 4.305; EA: 3.319; PP: 6.704; EE: 3.085 .

- Guernica. PNV: 3.577; HB: 2.335; PSOE: 704; EA: 1.367; PP: 371; EE: 434.

- Leiqueitito. PNV: 1.577; HB: 1.488; PSOE: 53; EA: 1.051; PP: 64; EE: 194. 
- Loyola. PNV: 3.822; HB: 1.570; PSOE: 2.045; EA: 660; PP: 614; EE: 1.085.

- Mungía. PNV: 2.972; HB: 951; PSOE: 420; EA: 675; PP: 274; EE: 293.

- Ondárroa. PNV: 2.173; HB: 2.352; PSOE: 178; EA: 618; PP: 297; EE: 291.

- Portugalete. PNV: 5.930; HB: 3.247; PSOE: 8.125; EA: 1.288; PP: 2.149; EE: 2.448 .

- Santurce. PNV: 5.511; HB: 3.912; PSOE: 6.208; EA: 1.302; PP: 1.441; EE: 1.833 .

- Sestao. PNV: 3.321; HB: 2.120; PSOE: 5.500; EA: 785; PP: 1.075; EE: 1.179.

- Valmaseda. PNV: 1.622; PSOE: 1.042; HB: 550; EA: 448; PP: 224; EE: 156.

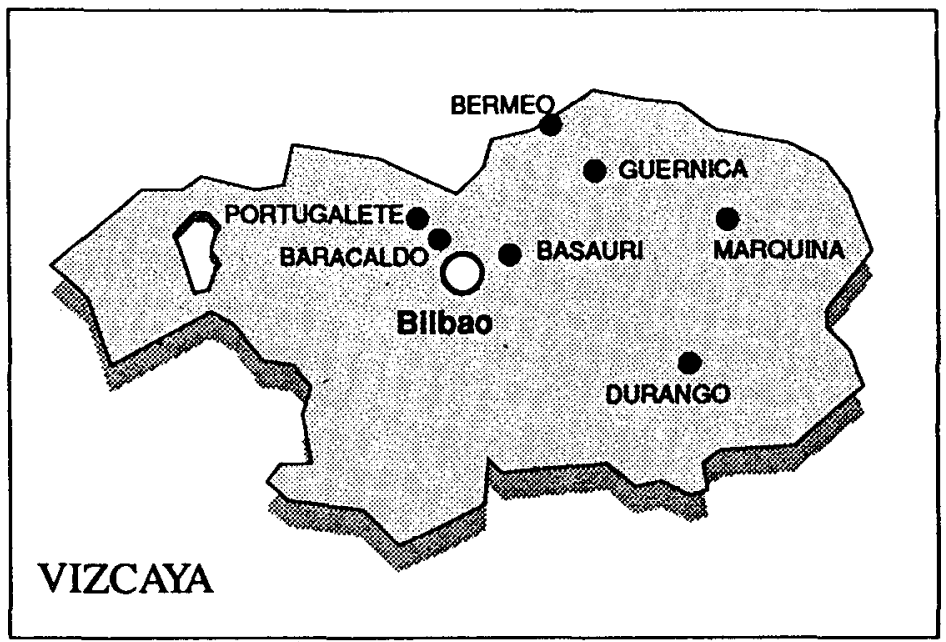


CUADRO 13

ÁLAVA, PUEBLO A PUEBLO

Estos son los votos obtenidos por los distintos partidos en las principales ciudades y pueblos de Álava, escrutado el 100 por 100:

- Vitoria. PSOE: 21.537; PNV: 17.299; UA: 11.326; HB: 10.328; PP: 10.168; EE: 6.638; EA: 6.313 .

- Alegria-Dulantzi. PNV: 199; PSOE: 102; UA: 36; HB: 32; PP: 31; EE: 15; EA: 18.

- Amurrìo. PNV: 1.425; PSOE: 802; UA: 64; HB: 877; PP: 432; EE: 337; EA: 981.

- Aramaio. PNV: 220; PSOE: 13; UA: 1; HB: 213; PP: 11; EE: 17; EA: 298.

- Artziniega. PNV: 282; PSOE: 41; UA: 4; HB: 125; PP: 116; EE: 33; EA: 29.

- Asparrena. PNV: 271; PSOE: 89; UA: 45; HB: 163; PP: 30; EE: 67; EA: 88.

- Ayala. PNV: 492; PSOE: 46; UA: 17; HB: 175; PP: 200; EE: 57; EA: 219.

- Bernedo. PNV: 92; PSOE: 9; UA: 25; HB: 14; PP: 31; EE: 6; EA: 4.

- Campezo. PNV: 236; PSOE: 74; UA: 18; HB: 50; PP: 131; EE: 17; EA: 70.

- Elciego. PSOE: 190; PNV: 51; UA: 32; HB: 23; PP: 91; EE: 26; EA: 73.

- Iruña de Oca. PSOE: 273; PNV: 165; PP: 108; HB: 62; UA: 63; EE: 39; EA: 22.

- Labastida. UA: 137; PSOE: 87; PNV: 78; HB: 64; PP: 84; EE: 12; EA: 12.

- Laguardia. PNV: 203; PSOE: 106; UA: 138; PP: 113; HB: 62; EE: 28; EA: 75.

- Lantarón. PNV: 170; PSOE: 29; UA: 81; HB: 31; PP; 60; EE: 10; EA: 10. 
- Legutiano. PNV: 207; HB: 143; PSOE: 71; EA: 111; UA: 15; PP: 20; EE: 20.

- Llodio. PNV: 2.761; PSOE: 2.019; HB: 2.305; UA: 105; PP: 917; EE: 660; EA: 1.088 .

- Salvatierra. PNV: 462; PSOE: 284; UA: 101; HB: 206; PP: 48; EE: 92; EA: 301.

- Urcaustaiz. PNV: 147; PSOE: 36; UA: 20; HB: 117; PP: 41; EE: 30; EA: 46.

- Valdegovia. PNV: 177; PSOE: 40; UA: 53; HB: 57; PP: 65; EE: 19; EA: 23.

- Zuya. PNV: 322; PSOE: 28; UA: 80; HB: 120; PP: 84; EE: 27; EA: 76.

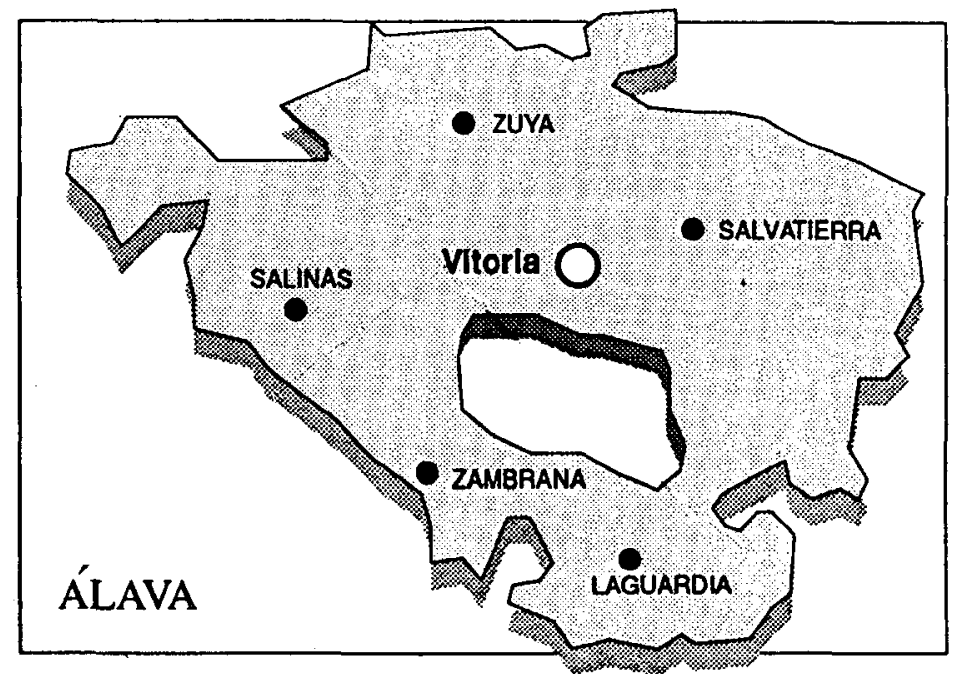




\section{Valoración}

Se confirma el multipartidismo del País Vasco, con una variación poco visible en el voto nacionalista, que baja dos escaños, y en el voto español, que sube otros dos en relación a la anterior consulta electoral; de todas formas se mantiene la proporción ${ }^{15}$, tradicional en todos los comicios, de dos tercios a un tercio, respectivamente ${ }^{16}$. La intención de voto, dirigida a respaldar una $u$ otra opción de gobierno, supone el cierre de un período de crispación política y social y un inicio de estabilidad con unos intereses diferentes. A continuación se irá examinando la evolución del voto ciudadano respecto a cada uno de los partidos en liza y la viabilidad de los futuros pactos.

a) El voto nacionalista PNV, EA, EE y HB, logra 50 escaños en el Parlamento de Vitoria, escaños que se reparten de la siguiente forma:

\section{Partido Nacionalista Vasco}

CUADRO 14

EVOLUCIÓN DEL VOTO DEL PNV ${ }^{17}$

(Porcentajes)

\begin{tabular}{|c|c|c|c|}
\hline 1980 & 1984 & 1986 & 1990 \\
\hline $37,58 \%$ & $42,01 \%$ & $23,64 \%$ & $28,5 \%$ \\
\hline
\end{tabular}

EI PNV logra un ascenso, 22 escaños frente a 17 de 1986, que, unido al retroceso seguido por EA y EE, hace del Partido Nacionalista Vasco el protagonista de la unificación del nacionalismo moderado y democrático,

15 Cotarelo, R., Diario 16 de los días $29-X-90$ y 5-X1-90.

16 Colomer, J., El País, 1-XI-90.

17 Los datos sobre evolución del voto de las distintas formaciones políticas desde 1980 proceden de $A B C, 29-X-1990$. 
superada la crisis sufrida en la consulta anterior. Lo dicho puede atribuirse al nuevo talante tolerante y pragmático del lendakari Ardanza, quien, ciertamente, ha logrado una disminución de los respaldos sociales a la violencia de ETA y un retroceso de los votos obtenidos por Herri Batasuna pese al mantenimiento de sus escaños ${ }^{18}$.

\section{Eusko Alkartasuna}

CUADRO 15

EVOLUCIÓN DEL VOTO DE EA

(Porcentajes)

\begin{tabular}{|c|c|}
\hline 1986 & 1990 \\
\hline $15,85 \%$ & $11,41 \%$ \\
\hline
\end{tabular}

El partido de Carlos Garaicoetxea sufre una considerable pérdida de votos -65.000- y de escaños, pasando de 13 en 1986 a nueve en los actuales comicios. Parece que la defensa radical del nacionalismo, a través de los medios democráticos, queda atrapada entre la oferta electoral del Partido Nacionalista Vasco y la posición anti-sistema de Herri Batasuna ${ }^{19}$; la pretensión post-electoral de EA de coaligarse con el PNV, en un intento de pervivencia, es clara muestra de lo dicho.

18 Pacto de Ajuria Enea entre los partidos democráticos de 13-V-88.

19 El Mundo, 29-X-90. 


\section{Euskadiko Ezquerra}

CUADRO 16

EVOLUCIÓN DEL VOTO DE EE

(Porcentajes)

\begin{tabular}{|c|c|c|c|}
\hline 1980 & 1984 & 1986 & 1990 \\
\hline $19,66 \%$ & $7,97 \%$ & $10,8 \%$ & $7,7 \%$ \\
\hline
\end{tabular}

Esta formación política pierde tres escaños y 45.000 votos, pasando de nueve representantes en 1986 a seis en la actualidad. La ambigüedad en la etapa de dirección de Kepa Aulestia, a caballo entre nacionalismo y socialismo ${ }^{20}$, ha perjudicado seriamente al partido de Bandrés, con un importante rechazo del electorado, inclinado hacia posiciones más nitidas y pragmáticas: o el PNV o el PSOE.

\section{Herri Batasuna}

\section{CUADRO 17}

EVOLUCIÓN DEL VOTO DE HB

(Porcentajes)

\begin{tabular}{|c|c|c|c|}
\hline 1980 & 1984 & 1986 & 1990 \\
\hline $14,1 \%$ & $23,07 \%$ & $22,04 \%$ & $18,36 \%$ \\
\hline
\end{tabular}

HB consolida sus 13 escaños, con un voto estable primado por la abstención. Sin embargo, la pérdida de 13.000 votos parece demostrar que 
esta organización política, que recibe su fuerza de la no comparecencia en las instituciones, ha logrado el techo electoral pese a la fidelidad de un electorado, de carácter militante, que se autoalimenta frente al aislacionismo ${ }^{21}$; en este sentido, si ha sido eficaz el pacto democrático, aunque no haya logrado todas sus pretensiones.

Pese al señalado Pacto de Ajuria Enea y la disminución de votantes señalada, HB continúa siendo la tercera fuerza parlamentaria del País Vasco y la primera en número de votos en su feudo de Guipúzcoa, empatada en escaños con el PNV; es en esta provincia donde la oferta de un gobierno abertzale ha logrado mayor respaldo, porque es esta provincia la que defiende más dogmáticamente la negociación con ETA, el reconocimiento del euskera como lengua nacional y la anexión de Navarra.

Si el giro de Herri Batasuna hacia su presencia en las instituciones se confirma, sus 13 escaños le presentan como una formación política a tener en cuenta; asi parece haberlo entendido el PNV al contar con este partido en sus conversaciones post-electorales, con los diferentes líderes políticos, decisión muy criticada en sectores españolistas ${ }^{22}$.

b) El voto españolista, PSOE, PP y UA, logra 25 escaños, dos más que en 1986, que se reparten de la siguiente forma:

\section{Partido Socialista de Euskadi}

CUADRO 18

EVOLUCIÓN DEL VOTO DEL PSE-PSOE

(Porcentajes)

\begin{tabular}{|c|c|c|c|}
\hline 1980 & 1984 & 1986 & 1990 \\
\hline $14,0 \%$ & $23,0 \%$ & $22,04 \%$ & $19,87 \%$ \\
\hline
\end{tabular}

Logra 16 escaños, lo que supone un retroceso respecto a los 19 logrados en 1984 y 1986; la causa quizá haya que buscarla en el desgaste

${ }_{21}$ Cotarelo, R., Diario 16, 5-XI-90.

22 Así, el Partido Popular manifiesta públicamente su indignación por estos contactos políticos. El País, Diario 16, 30-X-90. 
de este partido en el gobierno del Estado y en la alta abstención registrada en estos comicios, más sensible hacia los partidos gobernantes, como muestra de un cierto grado de desilusión. De todas formas se observa un reparto más equilibrado de sus votantes en las tres provincias, como respaldo paritario a su gestión en el Pais Vasco durante el último periodo.

\section{Partido Popular}

\section{CUADRO 19}

EVOLUCIÓN DEL VOTO DE AP/CP/PP

(Porcentajes)

\begin{tabular}{|c|c|c|c|}
\hline 1980 & 1984 & 1986 & 1990 \\
\hline $4,7 \%$ & $9,37 \%$ & $4,85 \%$ & $8,23 \%$ \\
\hline
\end{tabular}

Esta formación política logra un claro avance electoral, con seis escaños frente a los dos de 1986, lo que le permitirá tener grupo parlamentario propio en la Cámara Autonómica; es, junto al PNV, uno de los dos partidos que gana votos. Ello supone que ha desaparecido la prevención del electorado hacia la derecha españolista - hasta ahora demasiado antinacionalista- y que Vizcaya recupera la tradicional ideologia liberal-conservadora; el avance es más importante si se tienen en cuenta los 14.373 votos logrados por Unidad Alavesa, escisión muy reciente del Partido Popular.

La Unidad Alavesa logra tres escaños en la provincia de Álava que ningún sondeo adelantó; es la sorpresa de estas elecciones. Esta escisión del Partido Popular supo explotar los sentimientos de frustración de la sociedad alavesa frente a las otras dos provincias, ofertando la defensa de sus peculiaridades dentro de la propia personalidad vasca - «primero alaveses, luego vascos»-, amenazando con la segregación; es decir, manifiesta los conflictos interprovinciales dentro de la Comunidad. La prensa estatal critica abiertamente la tendencia tradicional de la derecha española a dividir sus fuerzas en grupúsculos de dudosa utilidad, renunciando a una 
unidad mucho más eficaz ${ }^{23}$. Ahora bien, frente a la frustración alavesa hay que recordar la representación desproporcionada que goza esta provincia en el Parlamento Vasco. El profesor De Pablo entiende que el pacto paritario sobre los 25 escaños lo forzó Álava por temor a quedar sojuzgada; el debate sobre su posible modificación ha provocado el voto a Unidad Alavesa ${ }^{24}$. Este partido utiliza frente a Euskadi igual amenaza que Euskadi frente al resto del Estado ${ }^{25}$; pese a ello, el futuro gobierno habrá de prestar atención a sus reivindicaciones.

\section{Centro Democrático y Social}

CUADRO 20

EVOLUCIÓN DEL VOTO DE UCD/CDS

(Porcentajes)

\begin{tabular}{|c|c|c|c|}
\hline 1980 & 1984 & 1986 & 1990 \\
\hline $8,41 \%$ & - & $3 \%$ & $0,65 \%$ \\
\hline
\end{tabular}

EI CDS no logra representación parlamentaria, siendo este tercer revés electoral ${ }^{26}$ una falta de respuesta del electorado a la ambigüedad de su oferta política; en esta consulta, el electorado vasco ha primado las opciones más claras, rechazando la confusión.

c) Las posibles coaliciones de gobierno

Ya se dijo cómo, desde la campaña electoral, el interés de esta consulta se centra en el respaldo ciudadano a una u otra posibilidad de pacto,

23 $A B C, 29-X-90$. Editorial.

${ }^{24}$ Catedrático de Historia de la Comunicación en la Universidad del País Vasco. El País, 4-XI-90.

25 Diario 16. «Opinión», 31-X-90.

26 Galicia, Andalucia, País Vasco. 
descontándose el que algún partido pueda lograr la mayoría absoluta. El Partido Nacionalista Vasco se encuentra, por tanto, ante una doble opción.

\section{c.1) : Coalición nacionalista}

Mayoría natural del nacionalismo democrático, PNV, EA y EE, que arrojaria la suma de 37 escaños, siendo 38 la mayoria absoluta; la aceptación de los votos de Herri Batasuna para paliar ese pequeño déficit supondria el convertirse en sus rehenes ${ }^{27}$, lo que es rechazado directamente por el partido vencedor ${ }^{28}$.

Eusko Alkartasuna y Euskadiko Ezquerra insisten, tras su descenso electoral, en formar esa mayoría natural que sería una posibilidad de remontar resultados. Sin embargo, este pacto no parece conveniente para el PNV, pues le obligaría a realizar una política nacionalista más radical que dificultaría su entendimiento con el Gobierno del Estado. De todas formas, parece que esta opción puede ser una baza importante para el PNV a la hora de negociar con el PSE, a quien se dirigen las preferencias del Partido Nacionalista desde el conocimiento de los resultados. La posibilidad de redondear los votos nacionalistas con una alianza con el Partido Popular no parece, en principio, muy deseable, pues crearia tensiones ante los distintos puntos de vista respecto a la autonomia, sin los beneficios que con el PSOE podrian obtener ${ }^{29}$.

\section{c.2) Coalición Partido Nacionalista Vasco-Partido Socialista}

La mayoría absoluta que tendría el pacto PNV-PSE demuestra el respaldo del electorado a esta coalición que, durante la legislatura 1986-90, contribuyó al inicio de la recuperación política y económica del país. El mayor número de votos del PNV, y la pérdida de 50.000 votos por el PSE, supone un nuevo equilibrio de poder en beneficio de aquél, que le permitirá dirigir las negociaciones y conseguir logros que compensen la renuncia a formar un gobierno nacionalista, bien visto por una gran parte del electorado.

27 Cotarelo, R., Diario 16, 5-XI-90.

28 Declaraciones de J. Arzallus recogidas por $A B C$ de 29-X-90.

29 El Pais, 1-XI-90. J. COLOMER. 
Esta coalición interesa al PNV para lograr el punto de encuentro con el Gobierno central, evitando la confrontación permanente en temas como el terrorismo, la organización de las fuerzas de seguridad y el iniciado debate sobre la autodeterminación; en igual sentido interesa al Partido Socialista estar presente en las soluciones que se tomen ${ }^{30} \mathrm{y}$, en otro ámbito, mantener buenas relaciones con el PNV de cara al Congreso de los Diputados, donde tiene una mayoría muy ajustada.

El Partido Socialista pretende -en contra de lo insinuado en la campaña electoral- formar parte de la coalición exclusivamente como partido representante de los vascos ${ }^{31} \sin$ que su posible negociación obligue al Gobierno central, punto crucial para los nacionalistas; de lo dicho se deduce que el interés del PNV por llegar a un entendimiento con los socialistas no se centra en sus afinidades ideológicas - tendría más con el Partido Popular- ni en su proyecto, distinto, para el País Vasco, sino en los beneficios que puede lograr al estar el PS en el Gobierno del Estado.

A la hora de redactar estas líneas siguen las difíciles negociaciones entre estas dos formaciones políticas, sin que se vea claramente la viabilidad de la coalición, o si el PNV tendrá que cambiar de rumbo y buscar alianzas entre la familia nacionalista, con los inconvenientes que se han señalado anteriormente.

Madrid, noviembre 1990

30 $A B C, 29-X-90$. «Opinión».

${ }^{31}$ Declaraciones de R. Jáuregui, recogidas por El País de 15-XI-90; ver nota 6 de este trabajo. 